\title{
Current quantization and fractal hierarchy in a driven repulsive lattice gas
}

\author{
Pietro Rotondo, ${ }^{1,2}$ Alessandro Luigi Sellerio, ${ }^{3}$ Pietro Glorioso, ${ }^{3}$ Sergio Caracciolo,, 3 \\ Marco Cosentino Lagomarsino, ${ }^{5,6}$ and Marco Gherardi ${ }^{3,5, *}$ \\ ${ }^{1}$ School of Physics and Astronomy, University of Nottingham, Nottingham NG7 2RD, United Kingdom \\ ${ }^{2}$ Centre for the Mathematics and Theoretical Physics of Quantum Non-equilibrium Systems, University of Nottingham, Nottingham NG7 \\ 2RD, United Kingdom \\ ${ }^{3}$ Dipartimento di Fisica, Università degli Studi di Milano, via Celoria 16, 20133 Milano, Italy \\ ${ }^{4}$ INFN Milano, via Celoria 16, 20133 Milano, Italy \\ ${ }^{5}$ Sorbonne Universités, UPMC Univ Paris 06, UMR 7238, Computational and Quantitative Biology, 5 Place Jussieu, 75005 Paris, France \\ ${ }^{6}$ CNRS, UMR 7238, Computational and Quantitative Biology, France
}

(Received 5 December 2016; revised manuscript received 20 August 2017; published 29 November 2017)

\begin{abstract}
Driven lattice gases are widely regarded as the paradigm of collective phenomena out of equilibrium. While such models are usually studied with nearest-neighbor interactions, many empirical driven systems are dominated by slowly decaying interactions such as dipole-dipole and Van der Waals forces. Motivated by this gap, we study the nonequilibrium stationary state of a driven lattice gas with slow-decayed repulsive interactions at zero temperature. By numerical and analytical calculations of the particle current as a function of the density and of the driving field, we identify (i) an abrupt breakdown transition between insulating and conducting states, (ii) current quantization into discrete phases where a finite current flows with infinite differential resistivity, and (iii) a fractal hierarchy of excitations, related to the Farey sequences of number theory. We argue that the origin of these effects is the competition between scales, which also causes the counterintuitive phenomenon that crystalline states can melt by increasing the density.
\end{abstract}

DOI: 10.1103/PhysRevE.96.052141

\section{INTRODUCTION}

In some areas of statistical physics-most notably for ferromagnetism, where the Ising model is the paradigmatic framework-classical coarse-grained theories have led to notable conceptual advances. For nonequilibrium transport, the reference models fall in the class of driven lattice gases (DLGs), which were originally introduced to capture collective phenomena out of equilibrium [1]. Such driven diffusive systems play an important role in nonequilibrium statistical physics [2], as well as providing models for various transport processes ranging from vehicular traffic [3] to biological transport [4-8]. At variance with equilibrium lattice gases, the DLG involves a driving field $E$, that causes particles to hop preferentially in one direction. The resulting steady states are characterized, for nonconservative fields (e.g., under periodic boundary conditions), by a net macroscopic current, i.e., they are genuinely nonequilibrium steady states. These models show a rich phenomenology, including nonequilibrium phase transitions and ordering phenomena such as pattern formation, self-organization, and morphogenesis.

In DLGs, the attention is usually restricted to nearestneighbor Hamiltonians [6,9-12]. However, in certain driven systems, longer-range interactions are an important defining ingredient. We particularly think of the context of hard condensed matter and ultracold atoms [13-16], where slowdecaying (especially repulsive) interactions are widespread, for instance, in dipolar fermions and Rydberg gases, where dipole-dipole and van der Waals forces dominate (e.g., the dynamical crystallization of a one-dimensional (1D) lattice gas made of Rydberg atoms has been observed experimentally

*Corresponding author: marco.gherardi@mi.infn.it
[17]). In this area, repulsive long-range lattice gases at equilibrium are sometimes used as reference models. In particular, they are invoked to explain the interesting phenomenology, such as devil's staircases and commensurability transitions, arising in experimental setups where a lattice spacing competes with the typical interparticle distance at fixed filling fraction $[18,19]$. These models (such as those introduced by Frenkel and Kontorova, Hubbard, and Bak) have a long history and a prominent place in the statistical mechanics literature, where they are regarded as prototypes of systems with competing interactions [20-25]. Despite the great interest met by the theoretical investigations of these models at equilibrium, explorations of their properties far from equilibrium are lacking. Notably, many charge transport phenomena in condensed matter are still debated or unexplained theoretically, highlighting the need for the study of such paradigmatic tractable models. Particularly challenging is the highly nonlinear behavior in current flow that signals anomalous transport in strongly correlated quantum many-body systems, which gives rise to complex phase diagrams [26]. Striking examples of electronic phases with anomalous emergent transport are the fractional quantum Hall effect (FQHE), the staircase of fixed-current phases in low-dimensional charge-density wave conductors (due to phase locking under ac bias voltage), resistive switching in Mott insulators, and the anomalous current-voltage characteristics in disordered films [27-31].

Here, we define and explore a one-dimensional DLG model with slowly decaying repulsive interactions. We fully characterize the behavior of the macroscopic current at varying field and density, by both direct simulation and analytical calculations. As we will show, this simple statistical mechanical model exhibits a rich phenomenology of anomalous transport, resembling some aspects of strongly interacting many-body systems out of equilibrium. 


\section{MODEL}

The system has $L$ sites and $N$ particles, fixed density $\rho=N / L$, and periodic boundary conditions. A configuration is specified by the set of occupation numbers $n_{i} \in\{1,0\}$ $(i=1, \ldots, L)$, with $n_{i}=1$ if the $i$ th site is occupied and $n_{i}=0$ otherwise. At equilibrium, the particles hop to nearestneighbor sites randomly with rate $w(\Delta H)=\min \left\{1, e^{-\beta \Delta H}\right\}$, where $\Delta H$ is the change in the energy function

$$
H=\sum_{i \neq j} V(|i-j|) n_{i} n_{j}
$$

due to the proposed jump, and $\beta$ is the inverse temperature. $V(x)$ is a repulsive convex potential; in particular dipolar and van der Waals interactions are characterized by a powerlaw decay $V(x) \sim 1 / x^{\alpha}$ with exponent $\alpha=3$ and $\alpha=6$, respectively. In the following, we discuss results obtained with $\alpha=3$. However, the phenomenology is robust, and does not depend on the exact functional form of the potential, provided that it is convex and vanishes at infinite distances [20].

Applying a constant field $E$ drives the system out of equilibrium. Owing to the periodic boundary conditions, $E$ is nonconservative, thus breaking detailed balance [2]. The microscopic effect of the drive is to bias jumps in one direction, as reflected by the modified hopping rates

$$
w(\Delta H+\ell E)=\min \left\{1, e^{-\beta(\Delta H+\ell E)}\right\},
$$

which take into account the work $\ell E$ against the field. Here, $\ell=-1$ and $\ell=+1$ correspond, respectively, to jumps along and opposite to $E$. We focus here on the zero-temperature case, where the rate is $w=0$ if $\Delta H+\ell E>0$ and $w=1$ otherwise.

Let us first briefly consider the zero-field stochastic dynamics, whereby energy is a nonincreasing function of time. At fixed density $\rho$, the configurations minimizing the energy are crystalline states with interparticle distances $\approx 1 / \rho$. However, the lattice introduces frustration (at densities different from $1 / q$, with $q \in \mathbb{N}$ ), forcing some of the interparticle distances to deviate from the average. In the ground state, described by Hubbard [20], the $n$th particle occupies the position

$$
x_{n}=\lfloor n / \rho\rfloor,
$$

where $\lfloor\cdot\rfloor$ denotes the integer part. A useful property of this state is the approximate equidistance of all $k$ th nearest neighbors. More precisely, $x_{n+k}-x_{n}$ is either equal to $r_{k}$ or to $r_{k}+1$, where $r_{k}=\lfloor k / \rho\rfloor$, for all $k>0$. Note that a Hubbard state at density $p / q$, with $p$ and $q$ coprime, has period $q$. Hence, it can only be constructed if the lattice size is a multiple of $q$. If this is the case, we refer to the state as supported by the lattice. The lattice size is set to $L=2520$, unless specified otherwise (this is the smallest size that supports all denominators up to $q=10$ ). Since the dynamics at $E=0$ is local, it is not a priori evident that the system should always reach the ground state from any starting configuration. We verified by extensive simulations that a disordered initial condition (a quench from temperature $T=\infty$ ) always evolves into the Hubbard crystal in a finite system. An example of a Hubbard ground state is shown in Fig. 1 for $\rho=3 / 10$.

We now turn to the nonequilibrium case $E \neq 0$, by simulation of the stochastic dynamics (2). If $N$ is the number

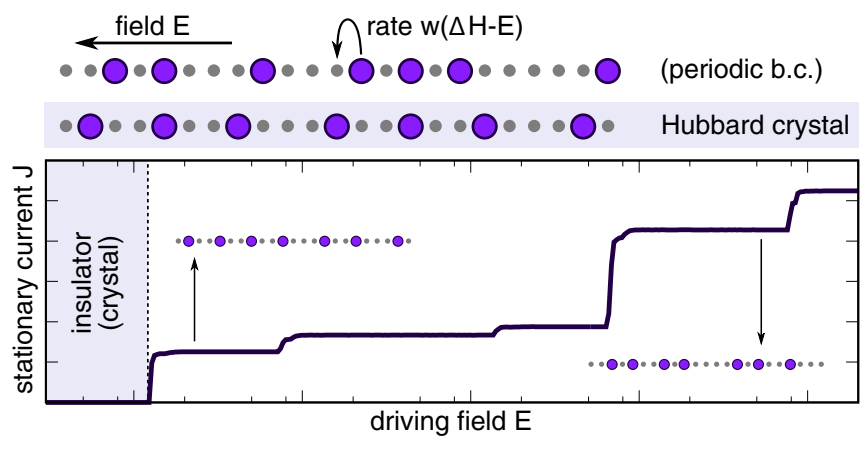

FIG. 1. Top: sketch of the model. Mutually exclusive particles hop to neighboring sites of a 1D lattice with periodic boundary conditions, with rates given by Eq. (2). Particles also feel pairwise slow-decayed repulsive interactions. Bottom: the stationary current (here at density $\rho=3 / 10$ ) as a function of the driving field is zero below a threshold, where the system is frozen in the Hubbard state, and anomalously quantized above. The arrows point to typical configurations at the corresponding $E$.

of particles in the system, a time step $(t \mapsto t+1)$ is realized by $N$ sequential updates. Each update consists of the choice of a random particle (uniformly on all particles) and a random neighboring site; if the site is empty, the particle is moved with probability $w$ given by (2). Here, the initial condition $\left\{x_{i}(0)\right\}$ is the Hubbard state, but we tested that a hot start at infinite temperature does not affect the results. The driving field is directed towards decreasing positions. The local current $j_{i+1, i}(t)$ is defined as the particle current across the bond $(i+1, i)$ between times $t$ and $t+1$, i.e., the number of particles jumping from site $i+1$ to site $i$ minus the number of particles jumping in the reverse direction. We study the behavior of the average stationary current, measured as

$$
J=\frac{1}{\left(T-T_{0}\right) L} \sum_{t=T_{0}}^{T} \sum_{i=1}^{L} j_{i+1, i}(t),
$$

where $T_{0}$ is a relaxation time (fixed $a$ posteriori to 100 times the fitted exponential autocorrelation time of $\left.\sum_{i} j_{i+1, i}\right)$ and $T=10^{6}$.

\section{RESULTS}

\section{A. Anomalous quantization of the macroscopic current}

Figure 1 shows the current $J$ as a function of the applied driving field (here for density $\rho=3 / 10$ ). At small driving fields, the system behaves as an insulator with zero stationary current, lying in the Hubbard ground state. At a threshold field $E_{\text {thr }}$ the insulator breaks down to a conducting state. A similar (but continuous) transition was found in a simple exclusion process with next-to-nearest-neighbour interactions at fixed density 1/2 [32]; here, owing to the longer range of the interaction, we find transitions for all densities. In the conducting phase $E>E_{\mathrm{thr}}$, transport is anomalous, in that the system preferentially supports a discrete spectrum of currents. Rapid changes in current and small near-Ohmic regions alternate with large plateaus where the differential conductivity $\sigma(E)=d J / d E$ is zero, and the current is independent of the driving field. 

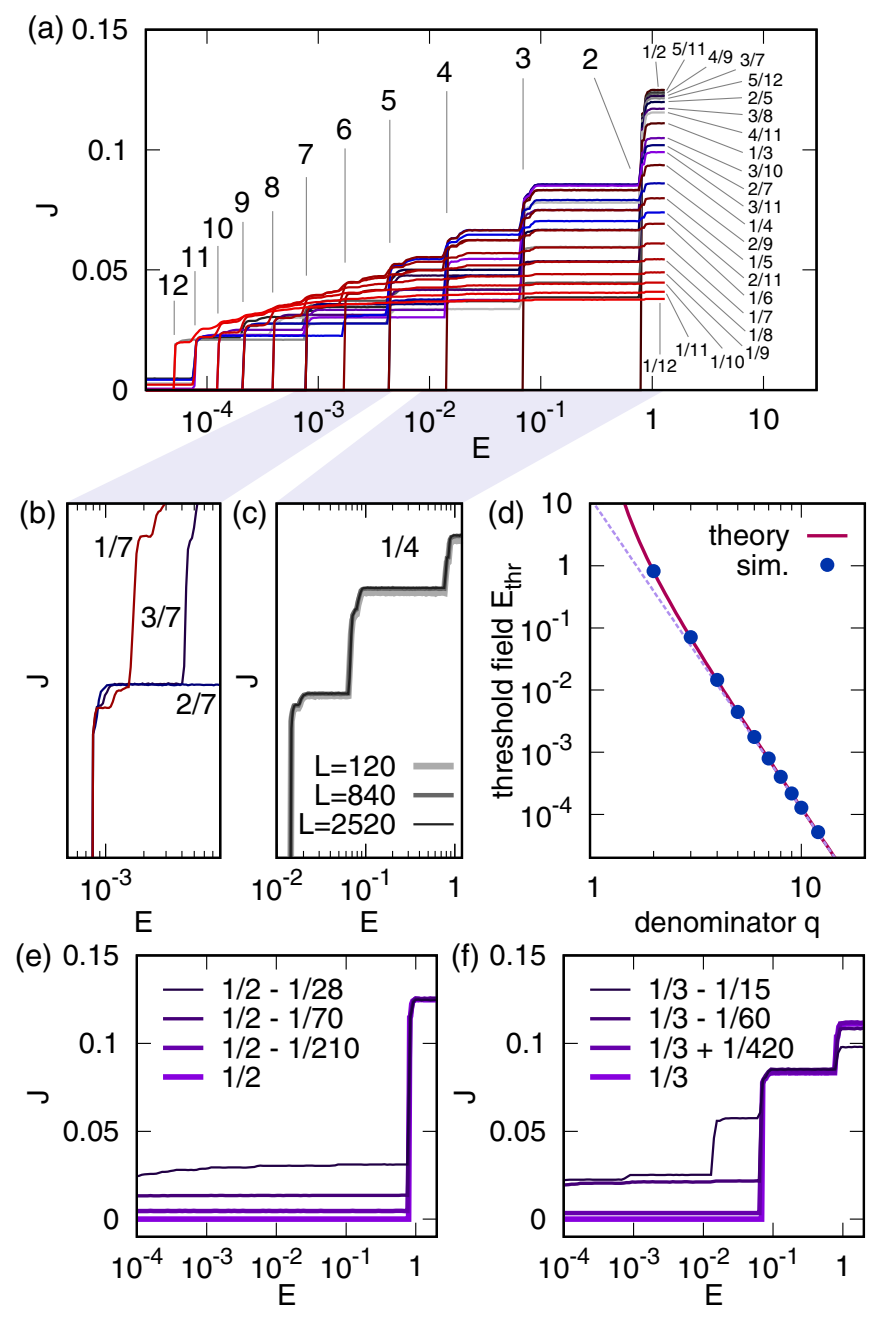

FIG. 2. Transitions arise at densities $p / q$, at threshold fields that only depend on the denominator. (a) Current versus driving field for various densities $p / q$ (numbers over the curves are the denominators $q$, with lines pinpointing the threshold fields). (b) A close-up showing only the curves for $q=7$. (c) The current at $\rho=1 / 4$ for increasing lattice sizes. (d) How the threshold field depends on the denominator: the circles are simulations, the solid line is the analytical formula Eq. (9), the dotted line is its asymptotic form $q^{-5}\left|\psi_{4}(1)\right| / 2$. (e), (f) Continuity of the current under variation of $\rho$, around $1 / 2$ in (e) (lines correspond to $\rho=13 / 28,17 / 35,52 / 105,1 / 2$ from top to bottom) and around $1 / 3$ in (f) (lines correspond to $\rho=4 / 15,19 / 60,47 / 140,1 / 3$ from top to bottom). Errors are smaller than the line widths.

The robustness of this phenomenology is visible in Fig. 2, which shows $J(E)$ for various densities $\rho \leqslant 1 / 2$. For values of $\rho$ above $1 / 2$, the plots are the same as those at $1-\rho$ because of the particle-hole symmetry $\left\{n_{i} \mapsto 1-n_{i}, E \mapsto-E\right\}$. The behavior of the current is robust in the sense that its features are independent of several details; in particular, Fig. 2 illustrates the following results. (i) The breakdown field $E_{\text {thr }}(\rho)$ only depends on the denominator $q$ of the density $\rho$, as we prove theoretically below. (ii) The current does not depend strongly on lattice size; in fact, the curves for $L=2520$ lie within the error bars of those at $L=840$. (iii) The locations of the main transitions between plateaus are largely independent of the density. The current at a given density $p / q$ appears to transition preferentially at or close to the threshold fields $E_{\text {thr }}\left(1 / q^{\prime}\right)$ of smaller denominators $q^{\prime}<q$. This is surprising, as the current-carrying excitations are expected to be different for different denominators. However, this property is a necessary requirement if the phenomenology is to be stable under small density changes, for instance when approximating $\rho=p / q$ on a lattice that does not support it. As an example, consider the currents for $q=11$. This denominator is not supported by $L=2520$, and $\rho=1 / 11 \approx 0.09091$ is rounded down to $229 / 2520 \approx 0.09087$. Despite the very different denominator, the current remains small below $E_{\text {thr }}(1 / 11)$ and becomes larger above. (iv) The current at the plateaus appears to be a continuous function of the density at fixed $E$. We checked this by testing several sequences of fractions converging to low-denominator ones, such as $1 / 2$ and $1 / 3$ [see Figs. 2(e), 2(f) for two examples]. Notice that a consequence of property (iii) above is that higher denominators entail curves with larger numbers of plateaux. Therefore, when considering a sequence with large denominators that converges to a simpler density, several consecutive plateaus must level out towards the same value. This behavior is evident, e.g., in Fig. 2(f). An important consequence of this continuous behavior is the fact that the extension of $J(E)$ to irrational values of $\rho$ is unique: it is possible to approximate the current-field curves for irrational densities with arbitrary precision in the thermodynamic limit $L \rightarrow \infty$.

Altogether, the foregoing observations show that the anomalous properties of $J(E)$ are not merely reflecting the microscopic details of the model, but realize a meaningful macroscopic phenomenology. Furthermore, they suggest that a continuum limit may be definable.

The breakdown field $E_{\mathrm{thr}}(\rho)$ can be evaluated analytically as follows. Its value must be equal to the energy difference between the crystalline ground state and the first excited state with a single defect. Let us consider the ground state $x_{n}=\lfloor n q / p\rfloor$ (with $p$ and $q$ coprime), and choose the $n$th particle, with $n$ a multiple of $p$. The $k$ th nearest neighbor on its left (right) will be a distance $y_{k}^{\mathrm{L}}\left(y_{k}^{\mathrm{R}}\right)$ from $x_{n}$. Moving the $n$th particle along the field (i.e., to the left) will change the interaction energy by a quantity $\Delta H=\sum_{k} \Delta H(k)$, where

$$
\Delta H(k)=V\left(y_{k}^{\mathrm{L}}-1\right)+V\left(y_{k}^{\mathrm{R}}+1\right)-V\left(y_{k}^{\mathrm{L}}\right)-V\left(y_{k}^{\mathrm{R}}\right)
$$

is the change of energy due to the interactions with the two $k$ th nearest neighbors. Since the state is periodic with period $q$, i.e., it repeats after $p$ particles, $y_{k}^{\mathrm{L}}=y_{k}^{\mathrm{R}}=m q$ whenever $k=m p, m \in \mathbb{N}$. The particles for which this holds will be called images of the one at $x_{n}$. Moving the $n$th particle to the left changes the interaction energy with its images by a quantity $\sum_{m} \Delta H(m p)$ that is computed below. Let us now focus on the particles between the first images, thus fixing $1 \leqslant k<p$. One has

$$
\begin{aligned}
& x_{n-k}=\lfloor(n-k) q / p\rfloor=n q / p-\lfloor k q / p-1\rfloor \\
& x_{n+k}=\lfloor(n+k) q / p\rfloor=n q / p+\lfloor k q / p\rfloor ;
\end{aligned}
$$

these relations are consequences of the fact that $n$ is a multiple of $p$, that $q$ and $p$ are coprime, and that $k$ is not a multiple of $p$. Hence, $y_{k}^{\mathrm{L}}=y_{k}^{\mathrm{R}}+1$; moreover, it is straightforward to check that this relation holds also if $k>p$, provided that again $k$ is not 
a multiple of $p$. Therefore, from Eq. (5), $\Delta H(k \neq m p)=0$, and finally

$$
\Delta H=\sum_{m} \Delta H(m p) .
$$

This energy variation holds if $n$ is a multiple of $p$, but it is the minimum over all particles. In fact, for all $n$ the image terms are the same as above, but all other terms in $\Delta H$ are non-negative, since $x_{n+k}-x_{n}$ is either $r_{k}$ or $r_{k}+1$. Finally, the energy gap is

$$
E_{\mathrm{thr}}(p / q)=\sum_{k=1}^{\infty}[V(q k+1)+V(q k-1)-2 V(q k)] .
$$

For the power-law potential $V(x)=1 /|x|^{\alpha}$ with integer $\alpha$, it evaluates to

$$
\begin{aligned}
E_{\mathrm{thr}}(p / q)= & \frac{(-1)^{\alpha}}{(\alpha-1) ! q^{\alpha}}\left[\psi_{\alpha-1}\left(1+q^{-1}\right) .\right. \\
& \left.+\psi_{\alpha-1}\left(1-q^{-1}\right)-2 \psi_{\alpha-1}(1)\right],
\end{aligned}
$$

which has the form of a finite-difference Laplacian of the polygamma function $\psi_{\alpha}(z)$, defined as the $(\alpha+1)$ th derivative of the logarithm of the gamma function $\Gamma(z)$.

The results of this calculation are in perfect agreement with the numerical simulations, as is shown in Fig. 2 for the potential $V(x)=1 /|x|^{3}$. The power-law asymptotic behavior $E_{\text {thr }} \approx q^{-5}\left|\psi_{4}(1)\right| / 2$, obtained from the analytical formula, is a good approximant already from $q=11$, with deviations below $2 \%$. The asymptotic behavior in the case $\alpha=2$ is $E_{\mathrm{thr}} \approx q^{-4} \pi^{4} / 15$. Due to the property (iii) discussed above, the analytical formula describes global features of the whole phase diagram, beyond the sole location of the breakdown transitions.

\section{B. Fractal hierarchy of excitations}

At any fixed density $\rho$, the current is a nondecreasing function of the field and reaches a maximum stationary value at large $E$. The saturation currents $J_{\max }(\rho)$ are those shown in Fig. 2 around $E=1$. A mean-field prediction, analogous to the standard one for simple exclusion processes, can be obtained by assuming that interactions become irrelevant at large fields, so that motion is constrained only by exclusion. The current is then proportional to the probability of finding an ordered particle-hole pair, i.e.,

$$
J_{\max }=\frac{\rho(1-\rho)}{2} .
$$

The mean-field formula perfectly captures the density dependence of $J_{\max }$, as can be seen in Fig. 3(c).

The saturation field $E_{\text {sat }}$, such that $J(\rho)=J_{\max }(\rho)$ for $E \geqslant$ $E_{\text {sat }}$, can be calculated exactly in the thermodynamic limit $L \rightarrow \infty$. Owing to the convexity of the potential, the largest energy difference in a single particle-hole exchange is attained when the particle has an infinite number of consecutive holes to its right and the hole has an infinite number of particles to its left, and is therefore $E_{\text {sat }}=V(1)=1$. Interestingly, the saturation field does not depend on the density, nor on the detailed form of the potential.
By lowering the driving field from $E_{\text {sat }}$, the density dependence of $J$ departs from the mean-field curve. Below $E_{\mathrm{thr}}(1 / 2)$, the system at half filling is in the insulator phase, and the stationary current must be zero, as it is for $\rho=0$. Therefore $J$ cannot be monotonic, and instead assumes a nearly parabolic shape [Figs. $3(\mathrm{~b}), 3(\mathrm{c})]$. Below $E_{\mathrm{thr}}(1 / 3)$, the system enters in the insulating phase for $\rho=1 / 3$ : the parabolic function splits into two daughter curves, with zeros in $\rho=\{0,1 / 2,1 / 3\}$. In the limit of small driving fields, this process generates iteratively a fractal hierarchy of branches. Between $E_{\mathrm{thr}}[1 /(q+1)]$ and $E_{\mathrm{thr}}(1 / q)$ the current has zeros for every $\rho=p / q^{\prime}$ such that $q^{\prime} \leqslant q$ (with $p$ and $q^{\prime}$ coprime). This sequence is called the Farey sequence $\mathcal{F}_{q^{\prime}}$ of order $q^{\prime}$ [33]. Since these are the only zeros, the plot of $J(\rho)$ realizes what is known as the Farey diagram in number theory, constructed by connecting consecutive fractions in the Farey sequences at all orders [see Fig. 3(d)].

It is interesting to note a connection between the Farey hierarchy described here and the fractal hierarchy of the crystalline ground states. It can be shown that the Hubbard state at $\rho=p / q$ can be constructed iteratively by considering the finite continued-fraction expansion

$$
\frac{p}{q}=\frac{1}{a_{1}+\frac{1}{a_{2}+\frac{1}{\ddots+\frac{1}{a_{\lambda}}},}}
$$

which is customarily denoted by $\left[0 ; a_{1}, a_{2}, \ldots, a_{\lambda}\right]$. At level 1 , the density $1 / a_{1}$ is simply realized by one particle followed by $a_{1}-1$ holes; let us call this block $X_{1}$ and denote it by (1) $(0)^{a_{1}-1}$. At level 2 , this arrangement gets corrected, by interposing a block $Y_{1}=(1)(0)^{a_{1}}$, which is longer and has smaller density, once every $a_{2}-1$ blocks of type $X_{1}$, hence obtaining a new block $X_{2}=\left(Y_{1}\right)\left(X_{1}\right)^{a_{1}-1}$. This procedure is repeated up to the final level $\lambda$ : at each level a larger-scale structure is specified (more details can be found in Ref. [20]). The foregoing construction gives rise to a hierarchy between rational densities, whereby $\rho^{\prime} \prec \rho\left(\rho^{\prime}\right.$ precedes $\left.\rho\right)$ whenever the continued fraction of $\rho$ starts with that of $\rho^{\prime}$, meaning that $\rho^{\prime}=\left[0 ; a_{1}^{\prime}, \ldots, a_{\lambda^{\prime}}^{\prime}\right]$ and $\rho=\left[0 ; a_{1}^{\prime}, \ldots, a_{\lambda^{\prime}}^{\prime}, \ldots, a_{\lambda}\right]$. This hierarchy is at the core of the fractal phase diagram that arises in the equilibrium $(E=0)$ model in the grand-canonical ensemble (first studied in Ref. [21]). The ground-state density as a function of chemical potential in that situation is a devil's staircase, a self-similar Cantor function with plateaus at every rational number; similar fractal layouts of transitions are often found in the presence of competing interactions or scales $[22,25,34]$. Interestingly, there exists a one-to-one correspondence between rational numbers and zigzag paths on the Farey diagram, starting from 0 and alternating between rightward and leftward jumps. This correspondence allows to easily construct the continued fraction of a rational number from the topology of its path (proofs of these results can be found in Refs. [35,36]). In this sense, the hierarchical layout of the excitations in our model realizes the out-of-equilibrium counterpart of the hierarchy of ground states.

Finally, we emphasize a counterintuitive aspect of $J(\rho)$, which is surprising from the point of view of driven diffusive 

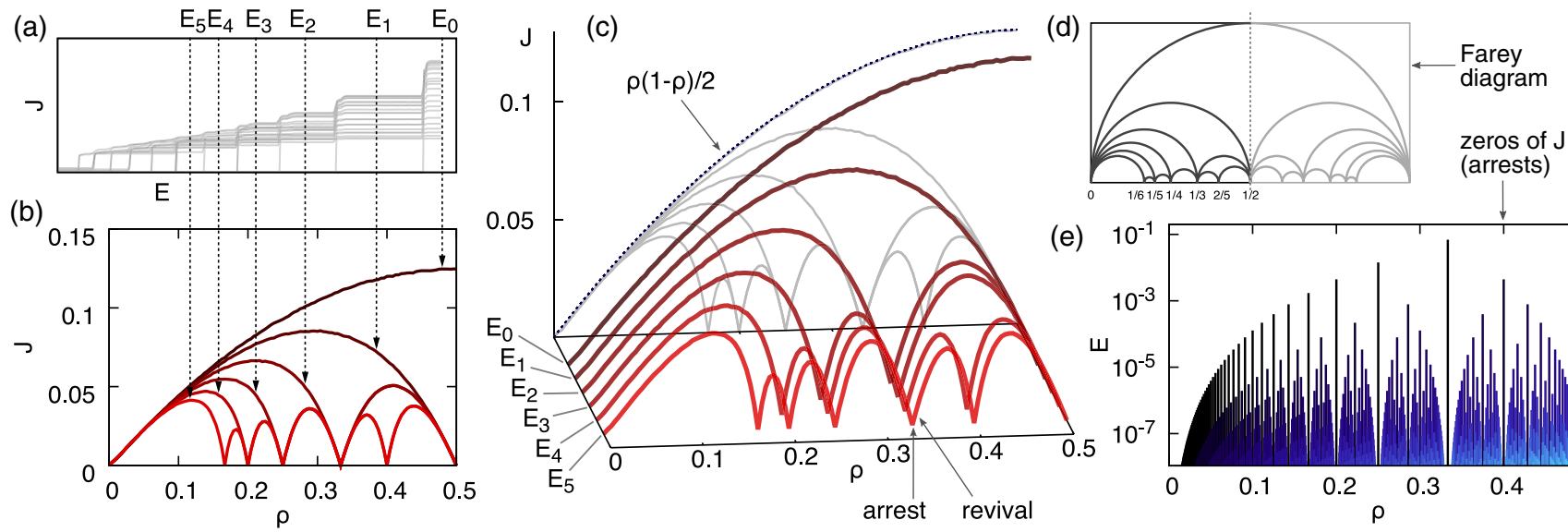

(e)

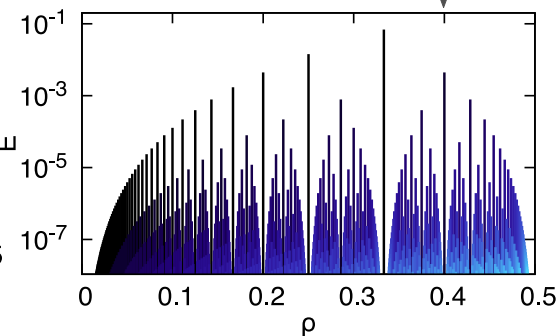

FIG. 3. The density dependence of the stationary current $J$ exposes a fractal hierarchy of excitations. Six values of the driving field $E$, chosen as shown in (a), which is the same plot as in Fig. 2(a), give the red solid curves in (b), (c). The dotted line in (c) is the mean-field formula for large fields $E>E_{\text {sat }}$. The fractal structure of the current-carrying excitations is captured by the Farey diagram of number theory, (d). As $E$ decreases, more and more densities crystallize. (e) shows all the zeros of $J(\rho)$ down to the threshold field for denominator $q=100$; lighter colors correspond to larger numerators $p$ (black is $p=1$ ).

systems. In classic short-range simple exclusion processes, the stationary current can become zero at a critical density, giving rise to an arrest (a traffic jam), which persists for all larger densities. By contrast, in the long-range DLG a jammed system can be revived by increasing the density. At fixed $E$, adding particles to an empty lattice of finite size first increases the current, then decreases it to an arrest where the system crystallizes. At this point, adding a single particle restores a nonzero current. Equation (9) gives the complete structure of the arrests with varying external field [Fig. 3(e)] up to a much larger detail than is attainable by simulations.

\section{DISCUSSION}

Driven diffusive systems are useful for both conceptual and applied reasons. On the conceptual side, they offer a broad class of statistical physics models that do not respect detailed balance. Thus, they provide examples of nonequilibrium stationary states, macroscopic and microscopic currents, pattern formation and nonequilibrium (and boundary-induced) phase transitions. On the applied side, they provide templates for models of specific systems, and clear interpretation tools that are possible only with models free of system-specific details. We believe that the DLG with slowly decaying repulsive interactions defined here has potential on both the fundamental and the applied side.

The most important conceptual contribution of the model defined here consists in the nontrivial transport properties related to the energy gaps between the configurations belonging to the nonequilibrium stationary states. The finite gap between the Hubbard ground state and the first excitation at fixed density is responsible for the insulator breakdown, as confirmed by the calculation of $E_{\mathrm{thr}}$. This (nonequilibrium) situation resembles the equilibrium grand-canonical case, where the crystalline ground states are incompressible, meaning that $\kappa=d \rho / d \mu$ is zero in a range of chemical potentials $\mu$. In this case, the differential conductivity $\sigma$ plays the role of the compressibility $\kappa$. This analogy is reinforced by the close similarity between Eq. (8) and the formula giving the stability interval $\Delta \mu$ of the ground states at density $p / q$, namely $\Delta \mu=$ $2 q \sum_{k} k[V(q k+1)+V(q k-1)-2 V(q k)]$ [21]. Notably, both $\Delta \mu$ and $E_{\text {thr }}$ depend only on the denominator of the particle density: this feature is responsible for the devil's staircase phase diagram at equilibrium and for the Farey hierarchy out of equilibrium.

On the more applied side, we believe that the repulsive driven lattice gas may play a role in the context of hardcondensed matter, as a simple paradigm of nonequilibrium transport showing a wealth of anomalous behavior. Indeed, no common interpretation tools are established for the peculiar transitions observed in driven quantum many-body systems. The correlated nature of the degrees of freedom is a major obstacle to theoretical advances in this field. The complex interplay between quantum effects, interactions, and macroscopic currents, together with the lack of natural perturbative parameters, have challenged the traditional descriptions in terms of Fermi liquids and crippled the development of a common theoretical framework for these systems [37-42]. Complementarily to other studies [38], our model suggests that repulsive interactions alone, in absence of quantum effects, are able to produce anomalous transport phenomena that are similar to those observed in some systems.

Whether this resemblance can be made more rigorous is an open question. The reduction to a classical kinetically constrained master equation may in some cases be approached rigorously, by starting from the quantum evolution in Lindblad form and integrating out the fast degrees of freedom [43]. Such approaches may reveal whether and to what extent the model defined here may capture the physics of a specific quantum system. Recently, the FQHE Hamiltonian has been mapped (in the thin-torus limit) to a classical equilibrium 1D lattice gas with repulsive long-range interactions, that is the grand-canonical version of our lattice gas at $E=0$, whereby the lattice is realized by the quantum states in the 
lowest Landau level [44]. The hierarchy of quasiparticles that emerges from the continued-fraction expansion is precisely the Haldane-Halperin hierarchy of the FQHE [45-47]. The fixed-current plateaus in our model correspond to currentcarrying excitations, whose layout as a function of $\rho$ in the $J$ versus $\rho$ plane (Fig. 3) parallels the FQHE phase diagram $[45,48]$. Here, the excitations realize the Farey hierarchy, somewhat complementarily to the Haldane-Halperin case. Also, the stability of a ground state under the external field $E$ is analogous to the stability of a FQHE state with respect to sample disorder, in the way the stability thresholds depend on the denominator of the density [45].

In conclusion, the repulsive driven lattice gas defined here shows how some highly nonlinear transport properties, closely resembling those in quantum many-body systems, can originate from a classical mechanism whose key ingredient are large-scale interactions. The crucial aspect leading to the observed behavior is the competition between the lattice scale and the inverse density. We stress that the phenomenology of the model is independent of the precise form of the potential (and thus in some sense universal), thus pointing to the importance for anomalous transport of the conflict between interactions and system-intrinsic length scales.

\section{ACKNOWLEDGMENTS}

We are grateful to B. Bassetti, P. Cicuta, J. P. Garrahan, and L. Moreschini for useful discussions, and to A. Vicini for providing computer time.
[1] S. Katz, J. L. Lebowitz, and H. Spohn, J. Stat. Phys. 34, 497 (1984).

[2] B. Schmittmann and R. Zia, in Statistical Mechanics of Driven Diffusive System, Phase Transitions and Critical Phenomena, Vol. 17, edited by C. Domb and J. L. Lebowitz (Academic Press, London, 1995), pp. 3-214.

[3] D. Chowdhury, L. Santen, and A. Schadschneider, Phys. Rep. 329, 199 (2000).

[4] D. Chowdhury, K. Nishinari, and A. Schadschneider, Phase Transitions 77, 601 (2004).

[5] D. Chowdhury, A. Schadschneider, and K. Nishinari, Phys. Life Rev. 2, 318 (2005).

[6] A. Parmeggiani, T. Franosch, and E. Frey, Phys. Rev. E 70, 046101 (2004).

[7] A. Parmeggiani, T. Franosch, and E. Frey, Phys. Rev. Lett. 90, 086601 (2003).

[8] R. Lipowsky, S. Klumpp, and T. M. Nieuwenhuizen, Phys. Rev. Lett. 87, 108101 (2001).

[9] T. Becker, K. Nelissen, B. Cleuren, B. Partoens, and C. van den Broeck, Phys. Rev. Lett. 111, 110601 (2013).

[10] J. Tailleur, J. Kurchan, and V. Lecomte, Phys. Rev. Lett. 99, 150602 (2007).

[11] S. Caracciolo, A. Gambassi, M. Gubinelli, and A. Pelissetto, J. Phys. A: Math. Gen. 36, L315 (2003).

[12] F. de los Santos, M. A. Muñoz, and P. L. Garrido, AIP Conf. Proc. 574, 149 (2001).

[13] E. Levi and I. Lesanovsky, New J. Phys. 16, 093053 (2014).

[14] B. Olmos, D. Yu, Y. Singh, F. Schreck, K. Bongs, and I. Lesanovsky, Phys. Rev. Lett. 110, 143602 (2013).

[15] L. Rademaker, Y. Pramudya, J. Zaanen, and V. Dobrosavljević, Phys. Rev. E 88, 032121 (2013).

[16] M. A. Załuska-Kotur and Z. W. Gortel, Phys. Rev. B 74, 045405 (2006).

[17] P. Schauß, J. Zeiher, T. Fukuhara, S. Hild, M. Cheneau, T. Macrì, T. Pohl, I. Bloch, and C. Gross, Science 347, 1455 (2015).

[18] Y. Zhang, J. Fan, J.-Q. Liang, J. Ma, G. Chen, S. Jia, and F. Nori, Sci. Rep. 5, 11510 (2015).

[19] A. Lauer, D. Muth, and M. Fleischhauer, New J. Phys. 14, 095009 (2012).

[20] J. Hubbard, Phys. Rev. B 17, 494 (1978).

[21] P. Bak and R. Bruinsma, Phys. Rev. Lett. 49, 249 (1982).
[22] P. Bak, Rep. Prog. Phys. 45, 587 (1982).

[23] U. Löw, V. J. Emery, K. Fabricius, and S. A. Kivelson, Phys. Rev. Lett. 72, 1918 (1994).

[24] O. Braun and Y. Kivshar, The Frenkel-Kontorova Model: Concepts, Methods, and Applications, Physics and Astronomy Online Library (Springer, Berlin, 2004).

[25] W. Selke, Phys. Rep. 170, 213 (1988).

[26] E. Dagotto, Science 309, 257 (2005).

[27] F. Nakamura, M. Sakaki, Y. Yamanaka, S. Tamaru, T. Suzuki, and Y. Maeno, Sci. Rep. 3, 2536 (2013).

[28] S. G. Zybtsev, V. Y. Pokrovskii, and S. V. Zaitsev-Zotov, Nat. Commun. 1, 85 (2010).

[29] L. Cario, C. Vaju, B. Corraze, V. Guiot, and E. Janod, Adv. Mater. 22, 5193 (2010).

[30] V. M. Vinokur, T. I. Baturina, M. V. Fistul, A. Y. Mironov, M. R. Baklanov, and C. Strunk, Nature (London) 452, 613 (2008).

[31] B. L. Altshuler, V. E. Kravtsov, I. V. Lerner, and I. L. Aleiner, Phys. Rev. Lett. 102, 176803 (2009).

[32] D. Helbing, D. Mukamel, and G. M. Schütz, Phys. Rev. Lett. 82, 10 (1999).

[33] G. H. Hardy and E. M. Wright, An Introduction to the Theory of Numbers, Oxford Science Publications (Clarendon Press, Oxford, 1979).

[34] J. J. Alonso, J.-P. Hovi, and H. J. Herrmann, Phys. Rev. E 58, 672 (1998).

[35] A. Hatcher, Topology of numbers, 2002, http://www.math.cornell.edu/ hatcher/ (accessed 2 Nov 2016).

[36] J. R. Goldman, Adv. Math. 72, 239 (1988).

[37] J. Eisert, M. Friesdorf, and C. Gogolin, Nature Phys. 11, 124 (2015).

[38] J. Jain and P. Anderson, Proc. Nat. Acad. Sci. USA 106, 9131 (2009).

[39] A. Georges, G. Kotliar, W. Krauth, and M. J. Rozenberg, Rev. Mod. Phys. 68, 13 (1996).

[40] T. Oka, R. Arita, and H. Aoki, Phys. Rev. Lett. 91, 066406 (2003).

[41] S. Krinner, M. Lebrat, D. Husmann, C. Grenier, J.-P. Brantut, and T. Esslinger, Proc. Natl. Acad. Sci. USA 113, 8144 (2016). 
[42] L. Moreschini, J. C. Johannsen, H. Berger, J. Denlinger, C. Jozwiak, E. Rotenberg, K. S. Kim, A. Bostwick, and M. Grioni, Phys. Rev. B 94, 081101 (2016).

[43] I. Lesanovsky and J. P. Garrahan, Phys. Rev. Lett. 111, 215305 (2013).

[44] P. Rotondo, L. G. Molinari, P. Ratti, and M. Gherardi, Phys. Rev. Lett. 116, 256803 (2016).
[45] E. J. Bergholtz, T. H. Hansson, M. Hermanns, and A. Karlhede, Phys. Rev. Lett. 99, 256803 (2007).

[46] F. D. M. Haldane, Phys. Rev. Lett. 51, 605 (1983).

[47] B. I. Halperin, Phys. Rev. Lett. 52, 1583 (1984).

[48] S. Kivelson, D.-H. Lee, and S.-C. Zhang, Phys. Rev. B 46, 2223 (1992). 